\title{
Belphégor
}

\section{Rapports de pouvoir : race, genre et nation dans la couverture montréalaise des JO de Berlin}

Camille Caron Belzile, Eve Léger-Bélanger, Alex Giroux, Marilou St-Pierre, Micheline Cambron and Dominique Marquis

\section{(2) OpenEdition}

Journals

Electronic version

URL: http://journals.openedition.org/belphegor/881

DOI: $10.4000 /$ belphegor.881

ISSN: 1499-7185

Publisher

LPCM

\section{Electronic reference}

Camille Caron Belzile, Eve Léger-Bélanger, Alex Giroux, Marilou St-Pierre, Micheline Cambron and Dominique Marquis, « Rapports de pouvoir : race, genre et nation dans la couverture montréalaise des JO de Berlin », Belphégor [Online], 15-1 | 2017, Online since 06 July 2017, connection on 20 April 2019. URL : http://journals.openedition.org/belphegor/881 ; DOI : 10.4000/belphegor.881

This text was automatically generated on 20 April 2019.

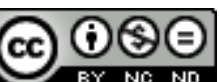

Belphégor est mis à disposition selon les termes de la Licence Creative Commons Attribution - Pas d'Utilisation Commerciale - Pas de Modification 4.0 International. 


\title{
Rapports de pouvoir : race, genre et nation dans la couverture montréalaise des JO de Berlin
}

\author{
Camille Caron Belzile, Eve Léger-Bélanger, Alex Giroux, Marilou St-Pierre, \\ Micheline Cambron and Dominique Marquis
}

1 Les Jeux olympiques, rassemblant pour deux semaines les meilleurs athlètes amateurs dans diverses disciplines, transportent avec eux toute une mythologie sportive liée à la célébration du corps discipliné, entrainé au maximum de ses capacités ${ }^{1}$. Cette mythologie trouve écho dans la couverture montréalaise des JO par la place accordée aux résultats des compétitions et aux nouveaux records établis par des athlètes qui semblent repousser de plus en plus loin les limites du corps humain. L'importance des résultats et des records, loin d'être l'apanage des Jeux plus récents, se retrouve au cœur de la couverture que donne la presse montréalaise des Jeux olympiques de Berlin². Ainsi, malgré l'ampleur des événements géopolitiques qui entourent les olympiades de l'été 1936, que ce soit la montée en force du fascisme, les relations tendues entre l'Allemagne hitlérienne et certaines nations dont la Pologne, la guerre civile espagnole qui fait rage, l'antisémitisme galopant et les menaces de boycott des Jeux, les médias montréalais, du moins ceux que nous avons étudiés à ce jour, ont braqué leur lorgnette sur l'événement sportif avant toute chose. Les listes des gagnants, les articles détaillant les compétitions de la veille ou les épreuves prochaines des athlètes canadiens sont les composantes qui ont dominé la couverture des JO.

2 Mais si important que soit l'aspect purement sportif des Jeux olympiques et le dépassement de soi des athlètes qui y participent, cette compétition internationale permet aussi de prendre la mesure d'un paradoxe inhérent à la pratique sportive : la nature intrinsèquement compétitive $d u$ sport, donc en principe ouverte à une libre concurrence, repose pourtant sur des hiérarchies préétablies ${ }^{3}$. Le sport est un marqueur de ségrégation selon la classe sociale, le sexe et la race, bref un marqueur de disparités comme l'illustre l'histoire de l'introduction des sports occidentaux dans les pays colonisés ${ }^{4}$. De plus, comme le donne à voir Suzanne Laberge ${ }^{5}$, le sport et les compétitions 
internationales ont été utilisés comme des outils d'exacerbation des rapports de pouvoir inégalitaires entre les nations, les classes sociales et les sexes, à cause de la disparité des ressources disponibles pour la pratique sportive selon les pays, et de leur distribution inégale selon les régions, les classes et les sexes.

Un second paradoxe, repérable dans la couverture médiatique des JO, est relatif à la promotion du caractère utopiste des Jeux. En effet, les dirigeants qui se sont succédés à la tête du CIO et des différentes instances décisionnelles placent les compétitions sous le signe de la paix et de la fraternité internationale. Ainsi, les JO modernes seraient en quelque sorte un modèle de neutralité politique ${ }^{6}$, argument qui sera d'ailleurs repris par certains pour justifier le maintien des Jeux de 1936. Pourtant, comme nous l'avons déjà souligné en ces pages, les JO sont tout, sauf apolitiques. Il n'est pas étonnant de voir s'esquisser, dans les discours populaires, un rapprochement symbolique entre les Jeux olympiques et une guerre sans armes, où chaque nation, avec ses ressources, tente de prouver sa valeur. En fait, les athlètes présents aux JO sont les porte-couleurs de leur pays, ils le représentent au même titre que des diplomates. Les Jeux olympiques constituent donc, pour les puissances mondiales, une manière d'afficher leur pouvoir et leur suprématie soit par le biais de performances mémorables soit à titre de pays hôte d'un événement sportif d'envergure. Le caractère politique des Jeux s'exprime également à travers les visées des nations participantes. Par exemple, Avery Brundage, président du comité olympique américain lors des Jeux de 1936, a admis devant certains proches qu'il visait, à travers les Jo de Berlin, à faire avancer sa vision de la démocratie « in the shape of a strong, successful Berlin Olympics against both Communism and the misguided politics of Eastern liberals in league with Jewish interest groups ${ }^{7}$ ». Rappelons de surcroit que les Jeux, qui se veulent un modèle d'ouverture, ne sont en fait pas véritablement ouverts à tous, ni à toutes 8 . La pratique sportive en général et les Jeux olympiques en particulier sont donc des lieux où s'entremêlent des discours contrastés, qui véhiculent des rapports de pouvoir qui ne sont pas toujours explicites, mais qui se trouvent manifestés dans le discours médiatique que diffusent les journaux. Selon Bernard Delforce, le journaliste est un acteur social qui donne «du sens au monde qui nous entoure ${ }^{9}$ ». Aussi, même lorsqu'un article apparait comme purement factuel, par exemple lorsqu'un journaliste relate les résultats d'une compétition de lancer du poids, nous sommes devant un acte de construction du sens. De la même façon, les décisions rédactionnelles, qui vont du choix des informations et du type d'article qui les véhiculera à leur mise en forme matérielle (format, titraille et choix d'un emplacement dans le journal), contribuent à la construction du sens. Dans le discours des journaux s'entremêlent donc des perspectives paradoxales, tant sur la nature compétitive des JO que sur l'identité des athlètes et leur statut social. Comment les journaux articulent-ils ces perspectives? La visibilité accordée dans les divers éléments de notre corpus ${ }^{10}$ à des constructions discursives liées à l'identité raciale, à l'identité genrée ou à l'identité nationale a retenu notre attention. Nous nous sommes demandé comment les éléments contribuant à l'élaboration de ces constructions rendent visibles les rapports de pouvoir qui traversent la société de l'entre-deux guerres, voire les problématisent.

\section{Récits identitaires sur la « race »}

4 Toutes sortes de commentaires ont circulé à propos du fait qu'Hitler aurait refusé de serrer la main du coureur américain Jesse Owens aux Jeux olympiques de Berlin parce que 
ce dernier était noir. En 1936, l'attitude d'Hitler face aux athlètes noirs fait couler de l'encre dans les journaux montréalais. Le chroniqueur Paul Parizeau du Canada affirme ainsi qu'Hitler agit différemment à l'égard des athlètes de race noire :

Fait plutôt insolite, chaque fois qu'un athlète de race noire a décroché les honneurs d'une épreuve, Hitler a brillé par son absence laquelle a été motivée par un appel urgent ou quelqu'excuse aussi diplomate... Voilà une vérité qui en dit beaucoup sur le mécontentement que doit ressentir le Fuehrer [sic] en constatant que les nègres américains ont enlevé à l'Allemagne cette suprématie à laquelle elle aurait pu aspirer sans eux ${ }^{11}$.

5 Une interprétation selon laquelle l'absence de poignée de main avec Owens serait un affront fait à la délégation américaine est aussi répandue, mais Baz O'Meara, chroniqueur au Montreal Daily Star, suggère que cela relève de la fabulation : "There are complaints that Hitler flouted the American delegation because he did not shake hands with Owens, but that viewpoint seems to be a strech of the imagination ${ }^{12}$ ». La question raciale est donc un enjeu à Montréal comme à Berlin. Dans les années trente, le comportement adopté à l'égard des Noirs est fort différent à Montréal de ce qu'on observe aux ÉtatsUnis, où sévit une ségrégation raciale inscrite dans la loi. En effet, l'absence de prohibition dans la province de Québec a fait du Montréal des années 20 une ville nocturne unique en Amérique du Nord, et les jazzmen noirs peuvent y exercer leur profession dans les clubs et les petites salles de spectacle, dans la mixité ${ }^{13}$. Cela ne signifie pas qu'il y ait absence de racisme mais cela montre clairement que les pratiques ségrégationnistes ne sont pas institutionnalisées. La question raciale est toutefois un sujet sensible comme en témoignent les extraits de chroniques reproduits ci-dessus.

Dans ce contexte, et compte tenu des débats antérieurs liés à la race des athlètes rappelons que le $\mathrm{CIO}$ a imposé à l'Allemagne une entente de non ségrégation à l'égard des athlètes ${ }^{14}$ - nous nous sommes demandé comment sont dépeints, dans les journaux montréalais, les athlètes qui ne sont pas blancs. Nous avons constaté, à notre grande surprise, que seule la race noire semblait faire l'objet d'un traitement différencié dans les textes, par une utilisation quasi systématique des épithètes " noir ", « nègre », « negro ", "colored» ou "coloré ", tandis que les athlètes asiatiques sont désignés par leurs qualités athlétiques plutôt que par la couleur de leur peau ou par d'autres particularités physiques, et que la question de l'identité des athlètes juifs est à peine effleurée ${ }^{15}$. Nous avons donc choisi d'analyser les figures discursives des deux grandes vedettes noires des Jeux selon les journaux montréalais, les coureurs américain et canadien Jesse Owens et Phil Edwards.

\section{Owens, la constellation noire - Edwards, le médecin de couleur}

7 Un enthousiasme généralisé à l'endroit de l'athlète Jesse Owens se dégage de la couverture des Jeux olympiques, comme le révèlent les titres dans les sections des sports : "La comète noire d'Amérique, le fameux Jesse Owens ${ }^{16}$ ", "Jesse Owens reste la vedette des Jeux olympiques, [sous-titre] la fameuse constellation de la piste américaine remporte, hier, son 3e titre dans le 300 mètres ${ }^{17}$ ", " Jesse Owens fait sensation aux Jeux ${ }^{18}$ ", "Owens is triple olympic winner ${ }^{19}$ ». Sans contredit, l'athlète est l'étoile des Jeux de Berlin avec ses quatre médailles d'or. Placé dans une altérité radicale grâce à des métaphores stellaires filant une allégorisation de la figure du héros du stade comme être de lumière et de vitesse, Owens est entouré d'une aura de merveilleux que confirme et amplifie l'oxymore «comète noire ». Certes, il ne ressemble pas aux figures canoniques 
des athlètes grecs évoqués dans le cérémonial du voyage de la flamme olympique puis de l'ouverture des JO : il est noir. Mais il échappe néanmoins à l'ordre humain pour devenir un dieu du stade.

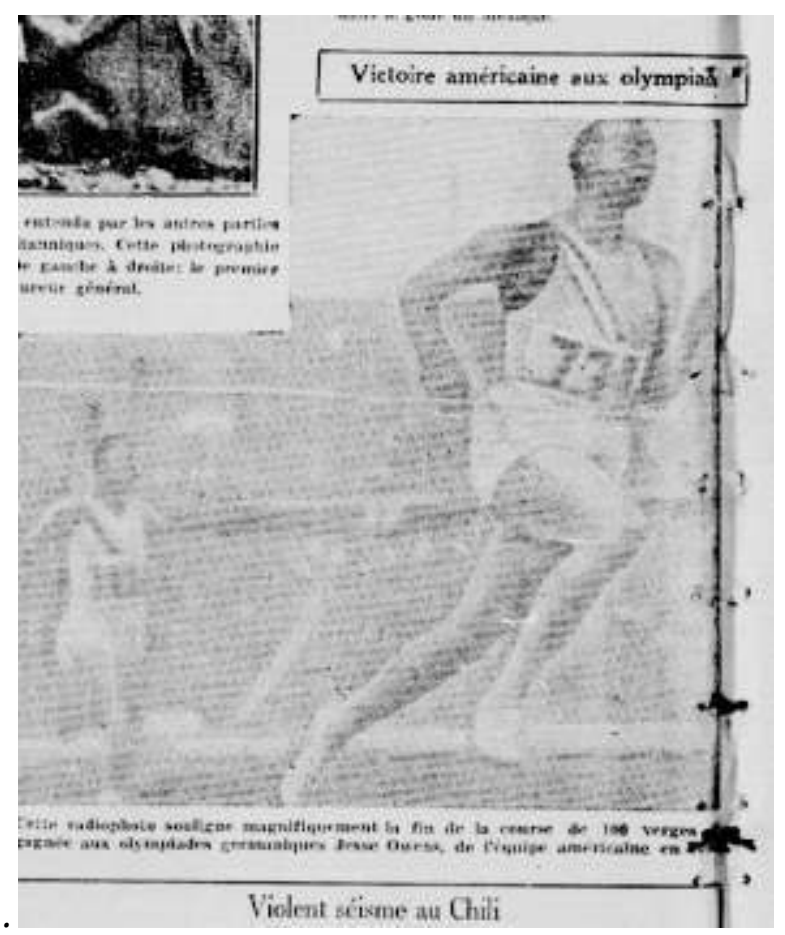

Figure 1 : Jesse Owens en action, «Victoire américaine aux olympiques », La Patrie, 5 août 1936, p. 12

Phil Edwards est quant à lui moins présent dans les grands titres, puisqu'il ne remporte qu'une seule médaille, de bronze. Lorsqu'il est question de lui, nous trouvons presque systématiquement son titre, "Dr», devant son nom, ou la mention "gradué de l'université McGill ». Il est par exemple question du « vaillant Dr Phil Edwards, gradué de McGill » et du «médecin de couleur, qui est un gradué de McGill ${ }^{20}$ ». Edwards est ainsi placé, dans le discours des journalistes, au sommet de la hiérarchie sociale montréalaise, là où sont les diplômés de McGill, alors l'université la plus prestigieuse au Canada.

9 Les journaux construisent donc deux figures opposées: l'une, celle d'Owens, est le résultat de l'inscription de la "comète noire " dans un autre monde, cosmique voire divin, l'autre, est le résultat de l'intégration d'Edwards à des cadres institutionnels et sociaux élitistes. Owens est propulsé dans l'altérité, Edwards ramené dans un ordre social où le statut professionnel l'emporte sur la couleur de la peau.

Néanmoins, ces figures sont parentes, leur couleur de peau étant constamment rappelée même s'ils sont présents quotidiennement dans les journaux. La désignation varie. Lorsqu'il est question des athlètes noirs dans les journaux francophones, il ne semble pas y avoir de distinction entre les termes «noir » et « de couleur »" Du côté anglophone, les termes «coloured» et «negro» dominent. Dans le Star et le Herald, le terme choisi à propos de l'athlète Phil Edwards, est « coloured » plutôt que " negro ». Certes, il est vrai que, au tournant du $\mathrm{xx}^{\mathrm{e}}$ siècle, dans un contexte d'autodésignation, le terme " colored » a été remplacé par celui de "negro » par la communauté afro-américaine elle-même ${ }^{22}$; il faut aussi faire la part de ce qui relève, dans les désignations, de la traduction directe des fils de presse par les journaux francophones. Il n'en reste pas moins que l'emploi du terme «negro ", ou "nègre ", par un blanc recèle toujours une connotation négative et 
que toute désignation raciale tend à enfermer celui qui en est affublé dans une définition stéréotypée de son identité, surtout à une époque où la ségrégation raciale est systémique dans certains pays. Le correspondant Henry McLemore constitue une exception puisqu'il ne mentionne jamais la couleur de peau des athlètes dont il parle. Cela rappelle au lecteur que la mise en apposition de la couleur de la peau n'est pas nécessaire au travail de nomination et que pour certains, seules comptent les actions des concurrents. La couverture des JO est ainsi l'occasion de pratiques discursives minoritaires.

\section{Le racisme comme objet}

11 Les textes provenant de fils de presse ne prennent pas position par rapport aux questions raciales. Soumis à une certaine censure, ils adoptent un ton factuel. Ils reprennent par exemple les paroles du ministre allemand de la propagande, Joseph Goebbels, qui affirme que sans ses athlètes noirs, la délégation américaine serait moins performante ${ }^{23}$, sans les commenter, ce qui laisse le lecteur libre d'interpréter ces propos soit comme un jugement de fait strictement comptable, soit comme une remarque insinuant que la nation américaine serait de moindre valeur parce qu'elle doit compter sur des Noirs, des inférieurs, pour gagner.

$12 \mathrm{Au}$ contraire, les chroniqueurs et signataires de textes prennent clairement position. La construction de figures d'athlètes noirs dans le discours invite à penser que les modalités de la nomination ne relèvent ainsi pas d'un simple automatisme, mais d'un choix conscient - du moins en partie - de leur part. Zotique Lespérance écrit par exemple que Brundage devra expliquer le renvoi des boxeurs noirs et l'exclusion des athlètes juifs américains $^{24}$. Au fil de sa réflexion, le chroniqueur s'interroge sur la ségrégation américaine :

La race noire a de nouveau dominé aux jeux olympiques et cela s'explique bien. Jesse Owens s'est révélé l'un des meilleurs athlètes de tous les temps. Owens caractérise aussi bien l'athlète de race noire. Les noirs deviennent de plus en plus menaçants et prédominants. Ces athlètes sont d'une endurance supérieure. On sent en eux une puissance, une résistance à l'effort qu'il est difficile d'abattre. Les épreuves sur piste et sur pelouse sont le seul domaine sportif où l'Américain traite d'égal à égal cette race pour laquelle il démontre, en tout autre circonstance, un véritable mépris. Owens, malgré sa race, est cependant devenu un grand héros américain [...] Les nègres de Harlem ne se sentiront plus d'orgueil et de joie comme au triomphe de Joe Louis... Depuis la Grande Guerre, l'on a constaté que seule une race forte pouvait être courageuse $e^{25}$.

13 Ainsi, selon Lespérance, les athlètes noirs états-uniens sont dominants, mais continuent à subir la discrimination. Le chroniqueur célèbre leurs performances et constate que le domaine des sports pourrait servir de tremplin vers une égalité sociale. Les chroniqueurs des journaux anglophones traitent quant à eux la stigmatisation associée au racisme en explicitant des principes moraux. Par exemple, Baz O'Meara dans le Montreal Daily Star pose sans détour que les authentiques amateurs de sport ne se soucient pas des questions raciales, mais des performances des athlètes, peu importe leur couleur de peau :

There is some anger among European competitors of continental strain at the supremacy of Owens \& Co. which manifested itself in cutting the editorial comment, but sportsmen who know no race, creed or color, but are keenly interested in performance have acclaimed the exploit of the dusky legion in the fashion known to sportdom for years ${ }^{26}$. 
Et il réitère sa prise de position les jours suivants, affirmant que la « race » des athlètes n'a pas d'importance.

Par-delà la langue et le parti politique, tous les journaux montréalais discutent des notions de race et de ségrégation, rendant lisibles ces enjeux. En effet, l'événement médiatique que constituent les Jo favorise l'explicitation des positions idéologiques et le travail discursif sur les identités comme on l'a vu dans la construction des figures d'Owens et d'Edwards issues de choix sémantiques qui nous ont révélé une sorte d'amenuisement des catégorisations raciales. La domination des athlètes noirs aux Jeux, principalement illustrée dans les journaux montréalais par les figures d'Owens et d'Edwards, semble contribuer à créer dans les journaux une discussion frontale sur le racisme et un questionnement sur les inégalités structurelles ${ }^{27}$. Mais sans racisme, et sans fascination pour les athlètes noirs, il n'y aurait pas de discussion sur la race dans les pages sportives.

\section{Rapports de pouvoirs genrés}

Les Jeux de Berlin ne sont pas les premiers à ouvrir leurs portes aux femmes. En effet, depuis $1900^{28}$, à l'occasion des Jeux de Paris, les femmes sont admises dans quelques sports $^{29}$. Lors des JO de Berlin, 331 athlètes féminines s'affrontent, soit $8,3 \%$ de tous les sportifs présents. Seuls quatre sports sont ouverts aux femmes ${ }^{30}$ pour un total de 15 compétitions. Il y a donc une marginalisation des athlètes féminines, qui d'une part sont numériquement beaucoup moins nombreuses, et d'autre part sont confinées à une pratique sportive peu diversifiée. Les infrastructures olympiques elles-mêmes témoignent du peu de considération à l'égard des femmes. Comme le rapporte La Patrie du 2 août 1936:

Les autorités olympiques allemandes sont encore préoccupées par le "problème féminin ». Ils (sic) n'ont pas encore déterminé s'ils (sic) se rendraient à la demande des Finlandais de faire venir une vieille femme au Village Olympique. Elle aurait charge de préparer les bains chauds des Finlandais. Mais voici la difficulté : le village fut construit pour loger 4,500 hommes... mais pas de femmes ${ }^{31}$.

La présence des femmes est donc très tôt liée à un problème logistique, alors qu'elles sont présentées comme devant "s'ajouter » à un village conçu et pensé par et pour des hommes. L'idée que les femmes appartiennent à un monde radicalement étranger à l'olympisme va de soi. De plus, il semble que la nourriture servie aux athlètes féminines dans les dortoirs qui leur sont octroyés soit insatisfaisante puisqu'elle est essentiellement composée d'aliments gras tels que des hamburgers et des frites ${ }^{32}$, un problème qui, selon les journaux, ne touche pas les hommes. Bref, si la présence des femmes est tolérée, elle repose sur un constant rappel de l'asymétrie entre féminin et masculin et sur la marginalisation qui en résulte. La couverture des JO dans la presse montréalaise ne dit pas autre chose.

Les «problèmes » liés à la participation féminine ne se limitent pas, comme nous le verrons dans les pages qui suivent, à l'aspect logistique des JO. Ils peuvent aussi être lus dans le cadrage médiatique ${ }^{33}$, c'est-à-dire dans l'orientation privilégiée par les journaux pour aborder le sujet "femme ", laquelle apparait comme une tentative de maintenir les frontières et la hiérarchisation entre masculin et féminin, au moment où le corps athlétique, exclusivement masculin, est mis sous tension par la participation des femmes. Ainsi en est-il du cas de l'américaine Eleanor Holm Jarrett, nageuse dont l'image de jeune 
femme blonde et jolie correspond parfaitement à la forme valorisée de féminité, jusqu'à ce qu'un écart de conduite vienne briser cette adéquation, et entraîne son expulsion par décision d'Avery Brundage ${ }^{34}$. Alors que l'écart à la norme féminine est sanctionné, les journaux montréalais rendent compte de l'affaire par la voix du mari de Holm Jarrett, et non par celle de la principale intéressée $e^{35}$, mettant en évidence les rapports hiérarchiques entre hommes et femmes. Mais cet exemple ne révèle qu'un aspect du discours sur les femmes dans les journaux montréalais.

\section{Marginalisation de la pratique sportive des femmes}

19 À l'image de la faible représentation des femmes dans l'espace olympique, les journaux montréalais analysés accordent une moindre place dans la couverture des Jeux aux exploits des athlètes féminines. Dans chacun des quatre périodiques étudiés en profondeur, nous retrouvons de manière récurrente un article principal sur les Jeux dans lequel on passe en revue les principaux gagnants de la journée, avec quelques détails sur les faits marquants des compétitions tel qu'un règlement contesté ou un espoir déçu. $\mathrm{Si}$ les compétitions féminines sont, en règle générale, évoquées dans cet article, le compte rendu qui en est fait ne s'étend que sur quelques lignes et se retrouve généralement placé vers la fin de la page ou, au mieux, en de très rares occasions, au tiers de l'article, jamais en début de texte.

De même, dans l'ensemble des rubriques consacrées au classement des médailles et aux résultats canadiens de la journée, les résultats des hommes sont systématiquement présentés avant ceux des femmes. On ne s'étonnera donc pas de ce que les gros titres portant sur une performance féminine soient très occasionnels. En fait, seulement deux grands titres, tous les deux dans le Star, font explicitement référence aux femmes ${ }^{36}$. Ce mécanisme médiatique de marginalisation des exploits féminins doit être replacé dans un contexte plus large de résistance face à l'intégration des femmes dans l'ensemble du dispositif du journal, alors qu'elles sont souvent confinées aux pages féminines. Dans le contexte spécifique des JO, les femmes sont exclues du discours journalistique autour de la figure olympique héroïque. À ce sujet, le traitement médiatique du cas Helen Stephens est éloquent. Nous y reviendrons en détail à la section suivante.

En plus de cette hiérarchisation de l'information qui marginalise les performances féminines, La Patrie, Le Canada et L'Illustration couvrent très discrètement la présence des femmes aux JO et n'accordent qu'une couverture restreinte aux performances féminines. Le Star se distingue en offrant une tribune spécifique au sport féminin, celle de Myrtle Cook, ancienne championne olympique, qui y tient une chronique quotidienne intitulée In the Women's Sportlight ${ }^{37}$. Selon la disposition habituelle, la chronique de Baz O'Meara est toujours placée en premier, suivie de celle de Cook à la page suivante. Cette dernière ne se retrouve donc pas sur la page consacrée aux JO. Cette page correspond à celle de la chronique de Baz O'Meara, qui pourtant consacre une proportion plus faible de son espace rédactionnel aux JO parce qu'il couvre également les sports professionnels masculins nord-américains. Les emplacements respectifs des deux chroniques ne sont pas sans suggérer une certaine hiérarchisation entre elles, mais il est difficile d'aller plus loin dans l'analyse du sens à attribuer à ce choix rédactionnel, puisque les chroniques de Cook visent spécifiquement à contrer la marginalisation du sport féminin. En effet, et malgré l'espace qui leur est dévolu dans le Star, nous pouvons conclure que globalement, les 
athlètes féminines sont marginalisées tant dans la forme que dans le fond des journaux montréalais.

\section{La réification des athlètes féminines}

L'une des pratiques discursives les plus significatives à cet égard, est le choix des descripteurs utilisés pour parler des femmes. Ces derniers témoignent de la conception normative qui, dans l'espace médiatique montréalais, travaille l'identité des femmes. Dans les journaux de 1936, il n'est pas rare d'utiliser des sobriquets ou des qualificatifs pour désigner les athlètes, autant les hommes que les femmes. En nous penchant sur les qualificatifs utilisés pour décrire les femmes, nous avons relevé : «jolie», "pretty», " jolie blonde", "jeune", " our pretty girls athletes", " our gallant relay quartette», "The pretty Nova Scotia girl», «La petite demoiselle», «la jolie Betty», «jolie jeune fille», etc. De tels épithètes et sobriquets, qui inscrivent la figure de la femme dans l'ordre esthétique dominent, s'opposant nettement aux descriptions des athlètes masculins. Les femmes ne sont pas rapides, agiles, fortes, ou braves, toutes qualités utilisées pour décrire les hommes, mais plutôt jolies, jeunes ou blondes. Ramenées à une nature qui ferait d'elles des objets de beauté, les athlètes féminines sont décrites à travers un lexique qui n'a rien à voir avec leurs performances sportives, et tout à voir avec des stéréotypes liés à la féminité. Ces épithètes témoignent d'un refus d'accorder au corps des femmes le statut de corps athlétique, le renvoyant plutôt à un statut passif, incarnant un objet de désir.

Ceci dit, notons que les photos, peu nombreuses, proposent un portrait plus diversifié des athlètes féminines. À l'exception de quelques photos de Holm Jarrett, montrée en contexte mondain et d'une photo montrant deux athlètes croquant une pomme dans une sorte d'allégorie biblique, nous voyons plutôt des femmes saisies durant une compétition ou bien posant avant ou après leur performance. La représentation visuelle montre des femmes en action, par exemple avec un casque de bain qui ne crée pas vraiment un effet « joli ». De plus, les légendes qui accompagnent ces photos sont généralement dépourvues 
de qualificatifs physiques. La féminité y est donc jouée de manière moins saillante que dans les textes eux-mêmes.

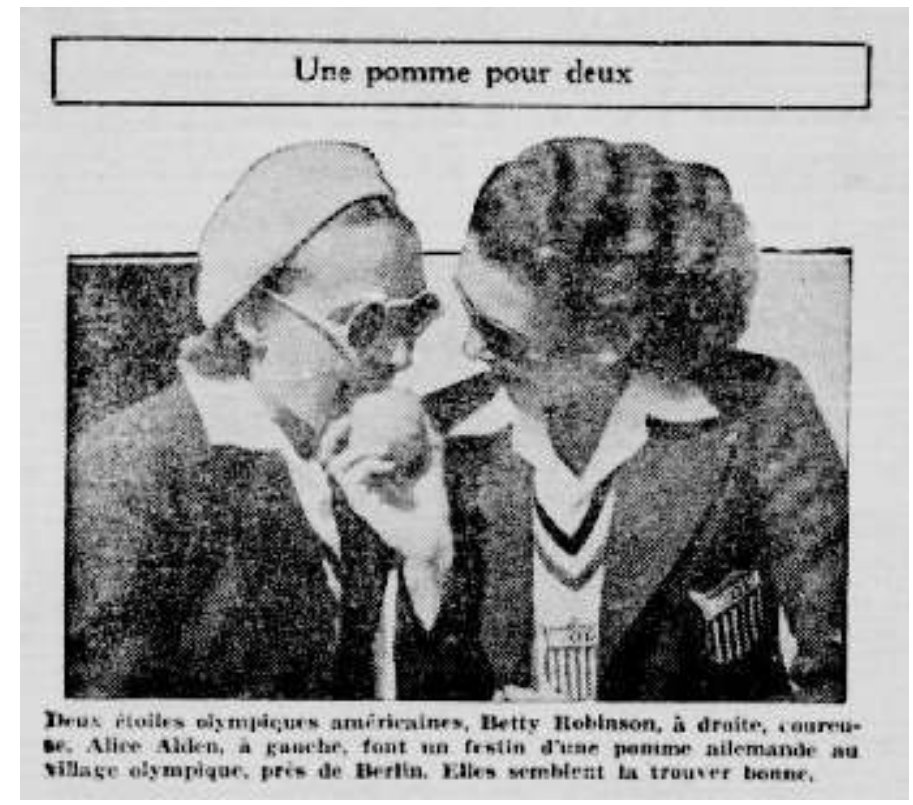

Figure 2: Image mondaine des athlètes américaines Betty Robinson et Allice Aiden, dans « Une pomme pour deux », La Patrie, 5 août 1936, p. 13

\section{De la construction de la féminité}

$\mathrm{Si}$, à l'exception du Star, les journaux montréalais se sont faits discrets quant à la présence des femmes, ils ont quand même consacré de l'espace à une polémique liée à la présence des femmes aux JO, soit la « féminité » contestée d'Helen Stephens.

Comme nous l'avons vu plus haut, les journaux montréalais contribuent à entretenir la division et la hiérarchisation entre le féminin et le masculin, entre autres par le biais de descripteurs, et par la présentation sexuée des résultats. Mais dans le cas de Stephens, la binarité ici évoquée est mise à mal, créant alors un récit duquel émergent les apories du système de genre. Le concept de système de genre renvoie à la division arbitraire et à la hiérarchisation des attributs, des comportements, des attitudes, en deux catégories, le masculin et le féminin, le premier étant placé en situation de dominance par rapport au second $^{38}$. Ainsi, à partir de certains signes dits biologiques, donc réputés neutres, un sexe, et corollairement une appartenance genrée, sont assignés aux individus, créant ainsi les combinaisons homme/masculin, femme/féminin. À partir de cette appartenance genrée se développe un ensemble d'attentes à l'endroit des individus, qui sont appelés à « performer le genre », pour reprendre le concept de Butler ${ }^{39}$, en respectant la catégorie à laquelle ils sont associés. Or, Helen Stephens vient bousculer un système bien établi, allant jusqu'à mettre en jeu le caractère supposément neutre du sexe. 


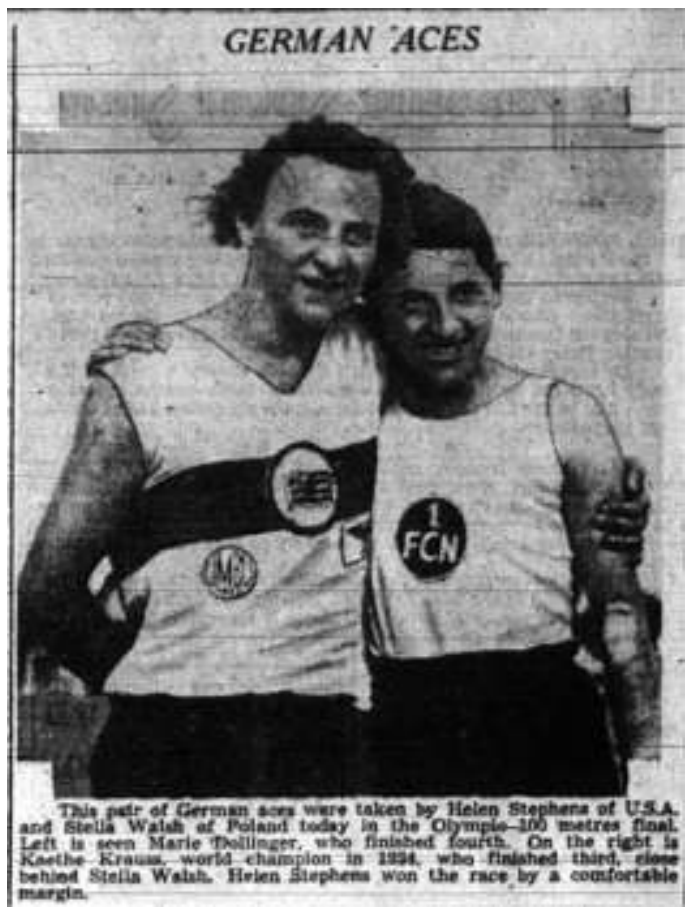

Figure 3: Helen Stephens et Stella Walsh, «German Aces », The Montreal Daily Star, 4 août 1936, p. 12

Pour résumer brièvement les événements, dès les premiers jours des compétitions, Helen Stephens s'impose sur la piste en battant la polonaise Stella Walsh en plus de briser le record mondial au sprint ${ }^{40}$. Dans les jours suivant cette victoire, un journal polonais, dont les propos sont relayés par les journaux montréalais, met en doute le sexe de la coureuse victorieuse, arguant que si Walsh avait concouru seulement avec des femmes, elle aurait remporté la victoire. À la suite de ces allégations, les journaux dévoilent que le comité olympique allemand avait déjà examiné Stephens avant de l'admettre aux Jo. Ainsi, « les juges, abasourdis par sa remarquable tenue, avaient conduit une inspection minutieuse pour établir sans aucun doute son sexe. ${ }^{41}$ " Si les allégations visant le sexe de Stephen s'ancrent en partie sur ses records « à un tel point sensationnels qu'ils égalent ceux des hommes ${ }^{42}$ ", ils trouvent également leur assise dans ses caractéristiques physiques. Par exemple, dans La Patrie, on insiste sur la pointure des chaussures de Stephens : du onze. De son côté, Myrtle Cook prend la défense de l'athlète, dans un commentaire direct et explicite :

She [Stephens] has a deep masculine voice it is true - she has big feet - a big strong body with a rippling muscles - but so have a lot of other girls who figure in the women's sport spotlight. Especially the deep voice. To offset these characteristics, Helen Stephens has shown a lot of girlish qualities that have been lacking in some of the less hefty competitors ${ }^{43}$. 


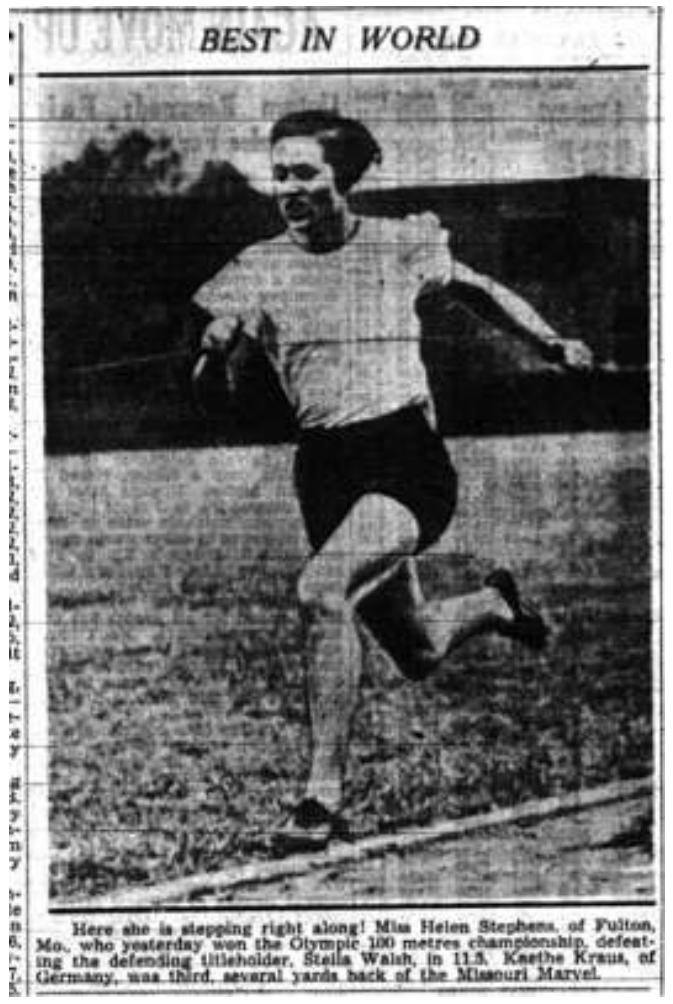

Figure 4: Helen Stephens, « Best in World », The Montreal Daily Star, 5 août 1936, p. 12

Donc, d'une part, les performances de Stephens sont jugées remarquables, mais elles sont tellement exceptionnelles pour une femme qu'il y a lieu de s'en méfier. Le système de genre fonctionne de telle manière que les femmes, dans un domaine réputé masculin comme le sport, ne sont pas supposées pouvoir égaler les hommes. D'autre part, comme le relève Cook, les caractéristiques physiques de Stephens, ces signes normalement neutres de l'appartenance au sexe "femme » ne concordent pas avec les autres signes envoyés : elle a de trop grands pieds, une voix trop grave et surtout, son corps possède des capacités normalement associées aux hommes, même si elle a des manières " féminines " (girlish). Le cas Stephens ébranle donc les certitudes quant à un sexe figé, immuable et neutre et met en lumière, à travers les pages des journaux et surtout celles du Star, le caractère social et construit à la fois de la catégorie «femme » et de ce que l'on appelle la « féminité » et, corollairement, de la catégorie « homme » et de la « masculinité ». Myrtle Cook le démontre lorsqu'elle met en lumière certaines caractéristiques physiques du champion du mille métrique, le Néo-Zélandais Jack Lovelock, qualifié de «slim, curly haired, handsome", dont la voix est "low and well modulated» et qu'on dit avoir un "shy girlish smile ${ }^{44}$ ", caractéristiques qui sont loin de coller à l'image de virilité des athlètes olympiques masculins. Toutefois, dans le cas de Lovelock, sa masculinité n'est en aucun cas mise en doute par le comité olympique ou par les journaux, ce qui nous rappelle le principe de hiérarchisation à l'œuvre dans le contexte sportif : un athlète qui bat tous les hommes ne peut être qu'un "vrai» homme, quitte à ce que ce double standard laisse entrevoir les apories de tout un système.

Cette hiérarchisation de l'espace sportif est également visible lorsque la Fédération sportive féminine internationale, qui gère depuis 1921 les activités internationales féminines, tombe sous la coupe de la Fédération internationale amateur, gérée entièrement par des hommes. Alors que la fédération féminine connaissait certaines 
tensions, «the men went far as to give the "Papa Will Spank " edict if the ladies body could not keep harmony within its ranks ${ }^{45}$ ", la hiérarchie établie entre les fédérations sportives masculine et féminine est explicite: si les femmes ne parviennent pas à s'entendre, les hommes s'en occuperont.

Ainsi, comme nous avons pu le constater, la présence des femmes aux JO émerge sur fond de réelles tentatives de domination de l'espace sportif olympique par le masculin, domination que redoublent les journaux par la place marginale laissée aux femmes athlètes dans les pages sportives. Toutefois, malgré les rapports de pouvoir saillants et lisibles dans le récit des JO de la presse montréalaise, la voix de Myrtle Cook $^{46}$ laisse filtrer un discours où pointent des tensions et une contestation du système genré, même si ce dernier semble immuable.

\section{La lutte des nations}

\section{La construction de la nation canadienne}

Sans surprise, la proportion des éléments des journaux mentionnant le Canada ou spécifiant qu'un ou des athlètes sont canadiens est importante dans le corpus. Même si cette proportion varie ${ }^{47}$, le Canada et ses athlètes sont des sujets de prédilection. On compte de trois à dix bandeaux portant sur le Canada dans chacun des journaux, de même que plusieurs sous-titres. Le sujet "canadien" est donc à l'avant-plan, malgré ses performances modestes. Les mots "Canada " ou "canadiens" coiffent de nombreuses listes de résultats ou de compétitions (60 sur 142) et près de la moitié des chroniques consacrées aux JO transmettent des informations sur les sujets "canadiens». Aucun journal ne se soustrait à la publication d'un «canadian outlook» comme le dit Myrtle $\mathrm{Cook}^{48}$. L'insistance des journaux montréalais à présenter les aspects canadiens des JO nous porte à croire que ceux-ci ont joué un rôle important dans la fabrication de la nation. Benedict Anderson a montré comment la presse écrite peut créer un sentiment d'appartenance dans une communauté de lecteurs en rendant possible le partage d'un ensemble de références communes, particulièrement grâce à des procédés d'ordre linguistique ${ }^{49}$. L'analyse montre que les périodiques montréalais contribuent ainsi à la construction d'une identité nationale canadienne.

31 Ainsi, on retrouve le procédé qu'Anderson désigne comme la «logoïsation » qui permet au lecteur d'imaginer la communauté nationale à travers des termes renvoyant à des symboles. Le drapeau ${ }^{50}$ et les " couleurs $~^{51} \mathrm{du}$ Canada sont ainsi mis à contribution, tout comme la feuille d'érable. Par exemple, un article de La Patrie parle du "Dominion », des "porte-couleurs du Canada", du fait de "porter les couleurs canadiennes" et des "délégués de la Feuille d'Érable " ${ }^{52}$. On retrouve dans le Star les mêmes symbolisations: «the wearers of the Maple Leaf ${ }^{53}$ » et «the Maple Leaf team ${ }^{54}$ ». Les descriptions que donnent les différents journaux du lever du drapeau canadien après la victoire de Frank Amyot témoignent de la force symbolique associée au drapeau et aux couleurs portées par les athlète ${ }^{55}:$ " the Canadian flag fluttering to the mast ${ }^{56}$ ", « les couleurs olympiques canadiennes ont flotté au haut du mât ${ }^{57}$ ", "L'emblème du Canada a été hissé au mât principal ${ }^{58} »$.

Un autre processus de «nationalisation » des JO est la dépersonnalisation des athlètes au profit de la mise en action de la nation. Ainsi, les journaux assimilent métonymiquement les athlètes canadiens et leurs exploits au Canada lui-même. Le Canada devient le 
principal acteur des JO : il «a été opposé à l'Allemagne ${ }^{59}$ " au relais, il «jouera sa deuxième partie [de basketball] dimanche ${ }^{60} \%$. On crédite de la sorte à la nation canadienne les résultats des compétitions : "What Canada did at Olympics Today ${ }^{61}$ ", "Une victoire olympique pour le Canada ${ }^{62}$ », «Le Canada et ses gloires olympiques ${ }^{63}$ ", "Les chances du Canada aujourd'hui ${ }^{64}$ », «Ce que le Canada a fait hier aux jeux ${ }^{65}$ ». Les membres de la nation doivent être fiers lorsque «le Canada fait belle figure... ${ }^{66}$ » et espérer quand " un espoir canadien ${ }^{67}$ ", ou "Canada" s main hope ${ }^{68}$ ", entre dans l'arène olympique. Les athlètes apparaissent en somme comme les "délégués de la feuille d'érable ${ }^{69} »$.

De plus, les différents chroniqueurs unissent les destinées du Canada, des athlètes canadiens et des lecteurs en mettant presque systématiquement à la première personne du pluriel les pronoms personnels et les déterminants, introduisant un sentiment de familiarité. L'emploi, pour désigner les athlètes, de termes familiers comme «boy », « lad ${ }^{70}$ », « little » ou « canuck ${ }^{71}$ ", de surnoms comme « Chuck » ou « Sleepy Sam », ou encore des seuls prénoms, renforce ce sentiment de familiarité. Tous ces procédés sont systématiquement utilisés par Myrtle Cook, qui unit ainsi la communauté canadienne à ses athlètes: "Our Canadian champion Hilda Cameron ", "Our young Canucks ", "We would like to see our Canadian four remain intact, on home ground ${ }^{72}$ ». L'emploi systématique de la première personne du pluriel permet au lectorat d'entrer, avec la chroniqueuse, dans le club sélect des Olympiens canadiens et, d'une certaine façon, de participer à la compétition : «Bleaker and bleaker becomes the canadian outlook on the natatorial front, just when we tought we really had something worth while up the Dominion sleeve, we take the well known cropper $^{73}$ ».

\section{Une définition du caractère national}

L'identification des athlètes à la nation canadienne est donc nette. Le discours journalistique esquisse en outre un idéal-type de la nation canadienne en insistant sur les qualités des athlètes. Si le médaillé d'or "Amyot proved that Canada still has men of brawn who can hold their own... ${ }^{74}$ ", c'est que ses qualités réactualisent des mythes fondateurs de la nation, présumés connus de tous ${ }^{75}$, qui reposent sur le fait qu'Amyot étant un canoteur, il évoque les explorateurs, les coureurs des bois et les marchands de fourrure. L'expression « to hold your own » suggère le courage, la ténacité et la résilience, toutes qualités nécessaires aux bâtisseurs du Canada. Amyot est considéré comme «l'homme qui n'a pas fléchii ${ }^{76}$ » et est donc donné comme modèle identitaire. Par ailleurs, les athlètes sont tous "jeunes", "brillants » et leurs "efforts " sont soulignés. Leurs qualités sont projetées, par l'éclat de leur performance, sur toute la «communauté imaginée » et soutiennent l'idée patriotique de l'importance du travail et du sacrifice pour la gloire et la pérennité de la nation, principalement à l'intention de la jeunesse, ce qui correspond aux propositions théoriques d'Anderson ${ }^{77}$.

Cependant, l'incarnation de ce modèle héroïque est à géométrie variable. La Patrie ne décerne pas pareil titre. Ailleurs, Amyot est présenté comme un héros, voire comme le seul héros national ${ }^{78}$. Quant à Edwards et Loaring, qui ont tous deux gagné le même nombre de points ${ }^{79}$, ils sont considérés variablement selon les journaux. Comme si Edwards n'était pas considéré de la même façon. Certes La Patrie le désigne comme le " porte-couleurs du Canada ${ }^{80}$ ", mais il se pourrait que pointent ici les limites du caractère inclusif de la nation. On sent, dans quelques textes, une mise en tension entre la couleur 
de la peau et l'idéal-type canadien. De la même façon, l'athlète féminine, décrite selon ses qualités esthétiques, particulièrement sa jeunesse et sa beauté, est exclue de l'idéal-type du Canadien vaillant. Sa présence permet toutefois que se décline un couple idéal canadien, composé d'un jeune homme vigoureux et courageux et d'une jeune femme jolie.

L'identité nationale n'exclut pas l'appartenance à une autre communauté. Les athlètes sont d'ailleurs presque systématiquement rattachés à une ville: «Frank Amyot d'Ottawa », «Edwards de Montréal », « Loaring de Windsor », « Hamilton's Betty » et Lee Orr de Vancouver. Unis sous l'étendard national, ils représentent aussi leurs communautés locales, plus spécifiquement leurs villes d'origine ou de résidence, lesquelles acquièrent une dimension identitaire. L'imaginaire national des lecteurs montréalais, par exemple, s'appuie ainsi sur une identité qu'ils partagent avec des citoyens lointains, qu'ils ne connaîtront, pour la plupart, jamais ${ }^{81}$. Comme l'illustre la description de la réception de Frank Amyot à son retour dans sa ville d'origine, Ottawa (la capitale nationale), l'identité nationale a pourtant préséance sur les autres identités ${ }^{82}$. Cette célébration revêt en effet un caractère pancanadien: les félicitations du Premier Ministre sont évoquées et c'est au parlement fédéral, un autre symbole canadien, qu'on demandera à Amyot "the dashing paddler to plant the Olympic tree ${ }^{83}$ ", croit Cook. De plus, la mairie d'Ottawa ne semble aucunement impliquée. Autre signe de cette préséance, O'Meara suggère même que le principe de représentativité par province soit écarté du processus de sélection des athlètes: "It does not matter where the good athletes come from. If it should happen that the best men in every sport come from one province or even one city - they should be selected ${ }^{84} "$, postulant de façon non équivoque que l'identité nationale l'emporte sur l'identité provinciale. Il ne semble pas admis qu'une identité nationale plus complexe, qui inclurait la langue par exemple, soit présente dans les journaux à l'occasion des JO. Comme on ne trouve qu'une seule mention d'un athlète canadien-français, celle d'un boxeur de Winnipeg qui a été défait dans les épreuves de sélection, et que l'absence de concurrents canadiens-français est quasi-totale, cela empêche de comprendre la place qui leur revient dans l'imaginaire national. Cependant, Georges Laferrière, de La Revue Moderne semble croire que ces derniers sont victimes de discrimination. Il est le seul commentateur de tout notre corpus qui discute de la participation des Canadiens-français aux Jeux ${ }^{85}$. 


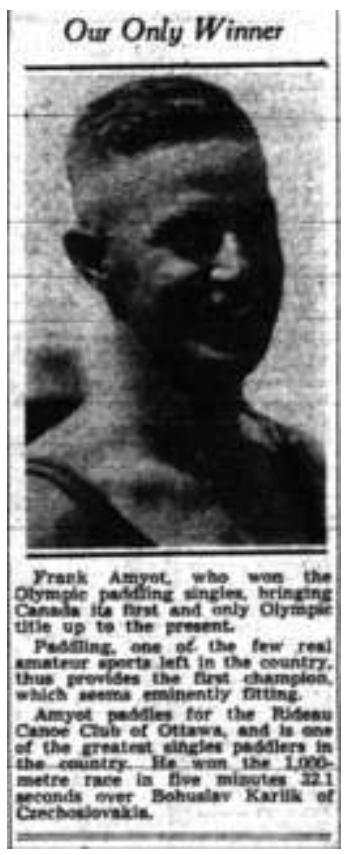

Figure 5: Frank Amyot, « Our Only Winner », The Montreal Daily Star, 10 août 1936, p. 19

La canadianisation de la couverture des Jeux olympiques est donc réalisée grâce à divers procédés discursifs. Au-delà de la quantité abondante d'informations « canadiennes », les athlètes des différentes villes et provinces canadiennes, leurs performances et leurs médailles sont « nationalisées » dans le discours, ils appartiennent donc à toute la nation. La définition de la nation canadienne qui en résulte est simple, rassembleuse et unificatrice. Elle est peu politisée, sinon dans une perspective restreinte à la gestion nationale de l'équipe olympique, comme le montre le très long article d'Horace Lavigne dans La Patrie du 9 août, et inscrit le sport olympique dans un continuum historique et national qui fait la part belle aux insuffisances ayant mené aux échecs des athlètes canadiens. 


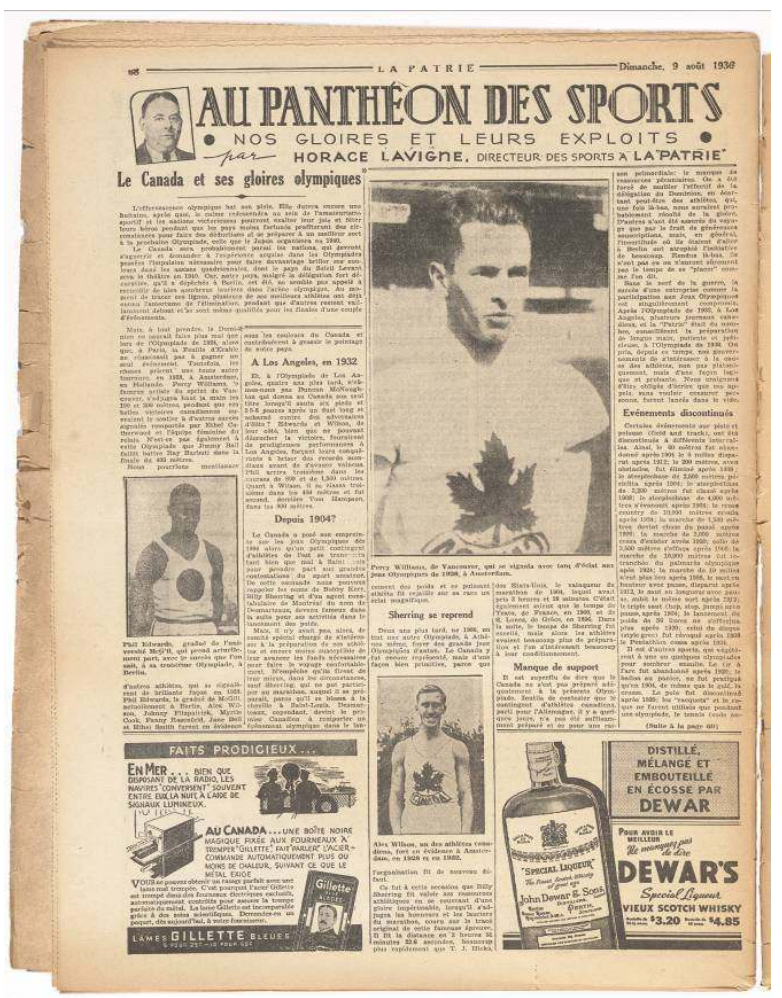

Figure 6 : Horace Lavigne, « Au panthéon des sports - Nos gloires et leurs exploits », La Patrie, 9 août 1936, p. 68

\section{Le sport et les relations entre Nation et État}

Le primat de la nation s'inscrit dans un discours qui définit les Jeux comme un concours entre nations. "L'effervescence olympique [qui] bat son plein ${ }^{86}$ met en scène une masse d'individus que l'on peut identifier à la nation. «[L] es nations victorieuses pourront exalter leur joie et fêter leur héros ", y compris de manière un peu fanatique. Myrtle Cook parle même de comportements assimilables à ce qu'elle nomme "adulation and heroworship ${ }^{87} »$. Elle prête de grandes attentes à cette «nation-public» dont les membres sont apparemment tous férus de sport : " as everyone on this side had expected ${ }^{88}$ ", « the folks at home demand winners to fawn upon and bask in their reflected glory ${ }^{89} \%$. Les chroniqueurs semblent ainsi avoir la conviction de comprendre et de refléter une opinion homogène.

Comme les performances canadiennes ne sont pas au rendez-vous, le public nous est décrit comme insatisfait. Myrtle Cook écrit: " John Public is not satisfied with the manner of selection ${ }^{90}$ ». O'Meara, parle du "[...] "Family Compact" that reigns in athletics», comparant le comité olympique responsable de la sélection à un groupe d'autocrates bien connu de l'histoire canadienne ${ }^{91}$. Le comité olympique « will have to talk loud and long to convince the fandom at large that our team was anything to brag over ${ }^{92} »$. Le Canadien moyen, «the man on the street ", qu'O'Meara considère particulièrement " au fait » et "dégoûté » à l'égard de l'issue de l'olympiade ${ }^{93}$, n'est donc pas dupe de la faiblesse du soutien du gouvernement fédéral. La presse dessine en creux un public très au fait des résultats de l'équipe olympique et qui réagit unanimement, de façon lucide, aux causes de l'échec. Convaincus qu'ils peuvent exercer une influence, et sur le lectorat, et sur les instances décisionnelles, les journalistes prétendent identifier les coupables. O’Meara, qui 
souhaite des changements lors de la préparation des prochains Jeux, affirme explicitement vouloir exercer une pression sur les autorités : «by keeping the public constantly reminded that the next Olympics should see Canada with a stronger team ${ }^{94}$ ». Pourtant, « après l'olympiade de 1932, plusieurs journaux canadiens, et La Patrie était du nombre, [avaient conseillé] la préparation de longue main, patiente et judicieuse ${ }^{95} \%$. Les politiciens auraient en somme sous-estimé la popularité des Olympiques. Ce manque de jugement se reflèterait même dans le déclenchement de la campagne électorale au Québec, reléguée, selon Elmer Ferguson à l'arrière-plan médiatique « with the Olympics [...] stealing the front pages ${ }^{96} »$. Les journalistes appellent donc l'État à se mettre à l'écoute de la nation.

Les solutions proposées pour les prochains Jeux passent invariablement dans les journaux par la participation du gouvernement et c'est donc lui qu'il faut convaincre que le succès sportif de la nation est sa responsabilité. "Don't you think it is about time that Canada woke up to its responsabilities? » déclare un «visiteur » du Star, cité longuement par Cook. Lavigne rappelle au gouvernement la récurrence des demandes déjà formulées avant les JO de 1936: «On pria [...] nos gouvernements de s'intéresser à la cause des athlètes [...] d'une façon logique et probante ». O'Meara relate des requêtes précises en faveur de la création d'une " commission du sport », appuyées sur l'argument selon lequel les «sport leaders all over Canada are failing to give the country [sufficient] athletic leadership and entertainment ${ }^{97}$ ". Les journalistes appuient donc leurs argumentations sur une conception de la démocratie dans laquelle l'État est considéré comme un levier essentiel dans la concrétisation des désirs de la nation eu égard aux Jeux Olympiques, même si ces désirs demeurent informes. Si, selon Lavigne, l'organisation des Jeux d'hiver de 1940 au Canada "stimulerait considérablement le sport ${ }^{98}$ ", il est possible, selon O'Meara, que cet événement émousse plutôt l'intérêt d'un public avide de spectacles de haut calibre et de gloire nationale plutôt que de conduire à la démocratisation de la pratique sportive par le développement de « recreational facilities and amateur sport ${ }^{99}$ ". L'implication de l'État dans le développement du sport apparait donc à la fois comme un outil propre à unifier la nation et à légitimer le pouvoir, comme on peut le voir pour les états-nations les plus performants, tels que l'Allemagne et les États-Unis.

\section{Identité canadienne et relations internationales}

41 Apparentés dans les journaux à un combat entre pays dans lequel les athlètes des étatsnations concurrents portent le poids des choix idéologiques de leurs dirigeants, les JO de Berlin mettent en relief les profondes divergences qui règnent entre les nations. $\mathrm{Si}$, d'entrée de jeu, La Patrie fait des prédictions pour le classement des nations ${ }^{100}$, McLemore et Ferguson jugent que l'importance de ce classement supplante totalement la doctrine officielle, "the essential, fighting well », qui valorise la participation individuelle. Ces deux journalistes souhaitent donc que leurs pays respectifs concentrent leurs efforts sur les épreuves où ils ont des chances de remporter des médailles, abandonnant les autres épreuves, et inévitablement les athlètes qui les pratiquent ${ }^{101}$. McLemore fait même preuve d'un certain fanatisme lorsque son appétit de médaille le conduit à déprécier les autres nations par l'usage de stéréotypes nationaux tournés en ridicule. "The Nazi version of hot $\operatorname{dog}^{102}$ » est pour lui la plus impressionnante chose qu'il ait vue en Allemagne et il semble trouver « delightful to see a worn-out and weary Finn [...] I had come to believe they never tired ${ }^{103}$ ». Certes, peut-être y a-t-il là un second degré, toutefois McLemore 
s'autorise l'expression, dans l'espace public, d'un chauvinisme impudent pouvant justifier l'ethnophobie de ses lecteurs.

De plus McLemore adopte une perspective uniquement américaine, quoiqu'il soit publié dans un journal montréalais et sa perception de la symbolique olympique - «The Olympic flame, symbolic of blazing competition and brilliant pageantry ${ }^{104}$ »- semble à l'opposé de la perspective majoritairement véhiculée dans les journaux étudiés. En effet, même si O'Meara comprend que l'« international rivalry sometimes overcomes the decorum ${ }^{105}$ ", il n'endosse pas les attitudes hostiles et agressives, qu'il dénonce à l'occasion, écrivant par exemple: "The Italians treated their opponent in good old tavern fashion ${ }^{106}$ ». En se prononçant sur les rivalités internationales, il expose implicitement sa vision du rôle international du Canada: "There is some anger among European competitors of continental strain at the supremacy of Owens \& Co. [...] The Italians, we imagine, did not like to see so many victories by the ebony hued race ${ }^{107}$ ». Dans cet énoncé où les modèles nationaux sont évoqués, se trouvent opposés l'attitude agressive des Italiens et des Allemands et le fair-play sportif qui semble aller de soi pour l'instance énonciative. Il n'est pas déraisonnable de dégager à partir de là une attitude proprement canadienne qui récuserait non seulement le manque de respect des adversaires mais aussi la vision belliqueuse des relations entre nations qui l'accompagne. De la même façon, lorsque Lespérance fait observer que «les Jeux olympiques pacifient certes les pays qui se préparent à une guerre mondiale ", et suggère que le Japon bridera ses intentions belliqueuses parce qu'il veut tenir chez lui les prochaines olympiades ${ }^{108}$, il pose (avec peut-être un brin d'ironie) comme allant de soi le désir de paix et le refus des antagonismes. Cook est la seule journaliste qui, au moment de son bilan, définit les JO comme permettant l'échange culturel et la camaraderie entre les pays en transcrivant en plusieurs langues, des adieux, des mots courtois, et faute de connaissance en certaines langues étrangères, un sourire comme langage universel ${ }^{109}$. Les JO semblent ainsi être l'occasion de promouvoir dans l'espace public une certaine façon de penser les relations internationales qui serait typiquement canadienne. En 1936, l'idée que véhiculent les médias montréalais à propos du rôle international du Canada, est celle d'une nation pacifique et ouverte, dont le patriotisme affable, peut malgré quelques tensions internes (à propos des Noirs, des femmes et des Canadiens français), désamorcer les tensions.

La nation canadienne, dont le citoyen idéal-typique est jeune, blanc, fort et courageux, accompagné d'une jolie femme, est conçue comme déployant un civisme et une politesse exemplaires dans l'arène internationale.

\section{Conclusion}

La couverture des Jeux olympiques de Berlin qu'offrent les journaux montréalais étudiés rend lisibles les rapports de pouvoir liés à la race et au genre. Certes, les discours reconduisent les stéréotypes de la période, parfois sans vergogne. Mais, parallèlement, les chroniqueurs formulent des critiques, ouvrent au débat des représentations qui semblent pourtant aller de soi. Bien sûr il y a des degrés dans la critique. Entre un Parizeau dont l'argumentation à l'encontre du racisme ouvert d'Hitler demeure implicite et une Myrtle Cook ou un Baz O'Meara, qui articulent rigoureusement leurs critiques à l'encontre des préjugés et en font le thème cardinal de leur couverture des JO, il y a une large marge. Néanmoins, nos analyses ont révélé que la remise en question des rapports de pouvoir n'était pas limitée aux journaux libéraux, ni aux journaux destinés à un public plus 
instruit. Elle est présente dans un journal comme La Patrie, qui se veut grand public, tout autant que dans le plus élitiste Star.

Cela dit, ces rapports de pouvoir reçoivent un traitement différencié dans les journaux. Alors que le caractère visible du racisme occasionné par les JO provoque des réactions parfois frontales, la construction de l'identité féminine dans le discours de la presse apparait le plus souvent comme allant de soi, comme en témoigne l'affaire Holm-Jarrett. En ce cas, l'ébranlement des évidences, la déconstruction des identités genrées est la quasi exclusivité de la seule chroniqueuse sportive à participer à la couverture des Jeux : Myrtle Cook. Il serait tentant de la considérer comme une marginale dont le discours ne porte pas et certes, l'existence de deux chroniques, l'une tenue par un homme, Baz O'Meara, l'autre par une femme, Myrtle Cook, indique bien la ségrégation sexuelle à l'œuvre. Ce serait toutefois oublier son statut de chroniqueuse établie, elle qui dispose d'une tribune bien visible et solidement implantée dans la durée. Contrairement à ce qui se passe en dehors des JO, Cook peut émettre ses commentaires sur le sport féminin dans un espace qui inclut les pratiques masculines, ce qui lui permet de déconstruire certains stéréotypes identitaires de manière efficace et convaincante.

Enfin, dans un contexte de mise en action de la nation, tel que les Jeux olympiques, le traitement médiatique des rapports de pouvoir qui met en jeu les femmes et les personnes de couleur peut être assimilé à un geste politique préparant une plus grande ouverture de l'espace public envers l'altérité. Mais il peut aussi être vu comme participant à un processus d'intégration des identités qui illustre - et peut-être légitime - la figure internationale que se construit au même moment le Canada comme instance pacifiste et conciliatrice. L'évolution des figures discursives reliées à la race, au sexe et à la nation des athlètes contribue à la mise en tension de l'identité collective imaginée mais aussi à l'établissement d'une image de marque. Les figures encore ambiguës qui apparaissent participent probablement à la mise en place inconsciente d'une sorte de tremplin discursif vers le décloisonnement du récit identitaire dominant dans lequel les minorités visent à légitimer leur place par le moyen de l'excellence sportive. Dans un contexte social aussi fortement marqué par la prolifération de discours et de gestes xénophobes, la prégnance de positions progressistes au cœur des journaux grand public montréalais permet de déceler l'importance de la ligne narrative de l'ouverture à l'Autre dans le récit identitaire de la communauté canadienne.

Loin d'être détaché des contingences du temps, tout récit collectif national est étroitement lié à l'existence d'un rapport concret entre des forces géopolitiques. En effet, dans le cadre des Jeux, événement-performance reposant sur la rivalité entre nations, la création d'une image nationale unifiée dans la diversité, où figurent femmes et personnes de couleur, donne du poids à ce qui paraît être un modèle culturel et politique canadien en voie d'achèvement. C'est en tout cas ce qu'illustre le montage photographique le plus saisissant de notre corpus, dans lequel le centre est occupé par le portrait d'un athlète blanc en pleine performance sportive, encadré de ceux de deux athlètes féminines et de deux athlètes noirs, dont l'un arbore la feuille d'érable sur son costume. 


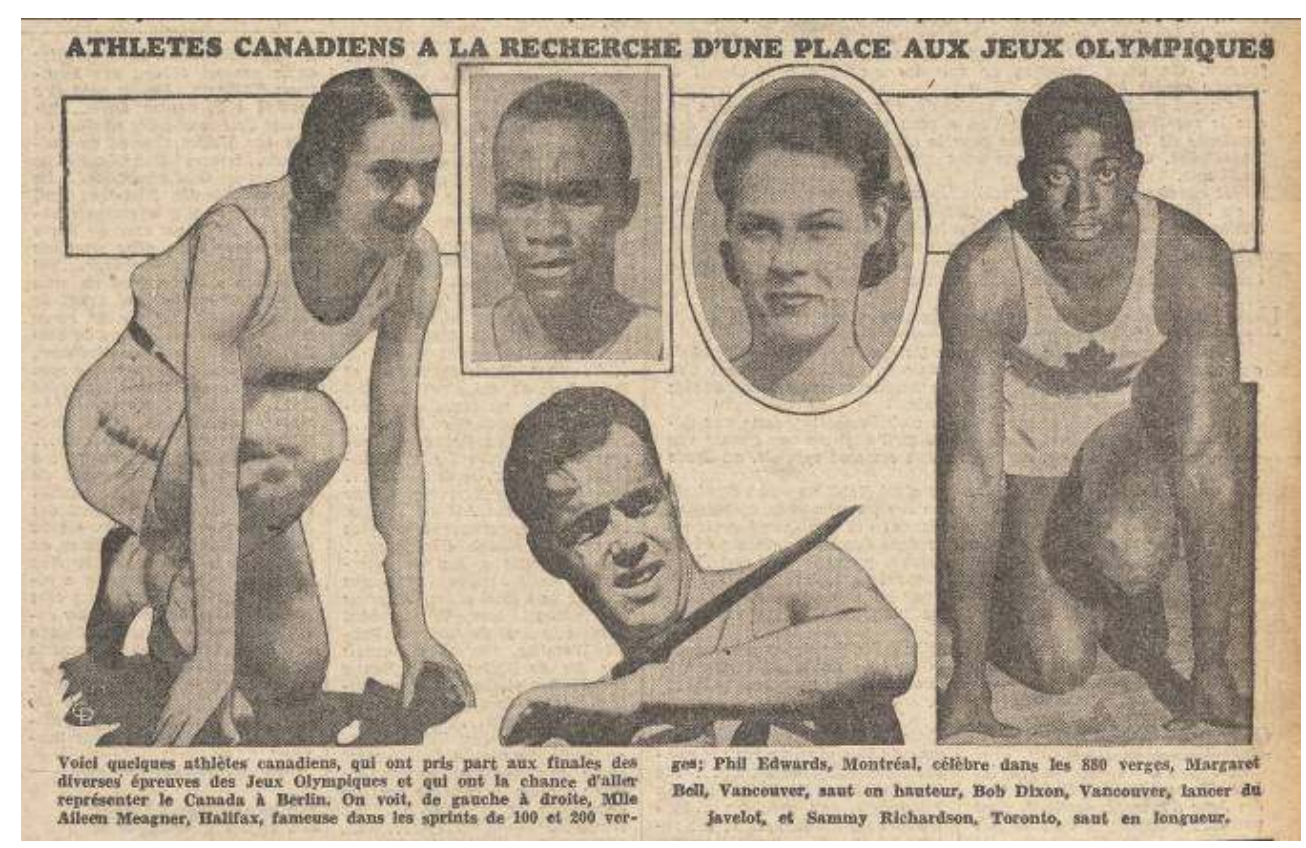

Figure 7 : «Athlètes canadiens à la recherche d'une place aux Jeux Olympiques », Le Petit journal, 2 août 1936, p. 56.

\section{NOTES}

1. David Rowe, «Modes of Sports Writing ", dans Journalism and Popular Culture, sous la dir. de P. Dahlgren et C. Sparks, London, Sage Publications, 1992, p. 96-112.

2. Voir dans ce numéro, «Les Jeux olympiques de Berlin dans l'arène médiatique montréalaise. Distance, censure et prise de parole ».

3. Ibid.

4. Michael A. Messner, Power at Play: Sports and the Problem of Masculinity, Boston, Beacon Press, 1992.

5. Suzanne Laberge, «Sports et activités physiques: modes d'aliénation et pratiques émancipatoires ", Sociologie et sociétés, vol. 27, n 1, 1995, p. 53-74.

6. Carolyn, Marvin, « Avery Brundage and American Participation in the 1936 Olympic Games », Journal of American Studies, Vol. 16, $\mathrm{n}^{\circ}$ 1, 1982, p.82.

7. Ibid., p.82-83.

8. Rappelons que Pierre de Coubertin, le père des Jeux olympiques modernes, ne pouvait imaginer la pertinence d'une « olympiade femelle » et s'opposait catégoriquement à la pratique sportive chez les femmes (d'ailleurs, il n'était pas plus ouvert à l'endroit des hommes de couleur). Ce qui n'empêcha pas les femmes d'accéder éventuellement aux Jeux olympiques, d'abord à titre non-officiel, puis officiel, et seulement en 1928 en ce qui a trait à l'athlétisme. « Les femmes dans le Mouvement olympique", Comité international olympique, 3 juin 2014, “ http:// www.olympic.org/Documents/Reference_documents_Factsheets/

La_femme_dans_le_Mouvement_Olympique.pdf. > (23 juin 2015) 
9. Bernard Delforce, «La responsabilité sociale du journaliste : donner du sens », Les Cahiers du journalisme, $\mathrm{n}^{\circ} 2,1996, \mathrm{p} .18$.

10. Nous avons ici travaillé de manière exclusive à partir des quatre journaux ayant fait l'objet d'une analyse systématique : La Patrie, L'Illustration nouvelle, le Montreal Star et Le Canada. Voir dans ce numéro « Les Jeux olympiques de Berlin dans l'arène médiatique montréalaise. Distance, censure et prise de parole».

11. Paul Parizeau, « Réflexions et commentaires », Le Canada, 12 août 1936, p. 11.

12. Baz O’Meara, « The Passing Sport Show », Montreal Daily Star, 4 août 1936, p.19.

13. Wilfred Emmerson Israel, "The Montreal Negro Community », Thèse de Ph. D., (sociologie), Université McGill, 1928.

14. Jean-Marie Brohm, 1936, Les Jeux olympiques à Berlin, André Versaille éditeur, 2008

15. Peu d'articles évoquent la polémique entourant l'exclusion d'athlètes juifs américains. Zotique Lespérance, " Nouvelles et commentaires sportifs », La Patrie, 13 août 1936, p. 21.

16. "Le Canada passe au cinquième rang de l'olympiade », La Patrie, 5 août 1936, p. 20.

17. "Jesse Owens reste la vedette des jeux Olympiques ", La Patrie, 6 août 1936, p. 20.

18. «Jesse Owens fait sensation aux Jeux» Le Canada, 6 août 1936, p. 11.

19. "Owens is triple olympic winner », The Montreal Daily Star, 5 août 1936, p. 19.

20. « Edwards se qualifie pour la finale des 800 mètres », La Patrie, 4 août 1936, p. 20.

21. À quelques reprises, le terme «nègre » est employé, mais ces occurrences sont tout de même moins nombreuses que celles des deux premiers termes et le mot semble employé sans connotation péjorative particulière.

22. Tom W. Smith, «Changing Racial Labels : From "Colored" to "Negro" to "Black" to "African American" ", Public Opinion Quarterly, vol. 56, 1992, p. 496-514.

23. On trouve trace de cette citation de Goebbels dans plusieurs journaux. Par exemple, «Goebbels newspapers about the colored athletes with the American team will hardly make for international amity " dans Baz O'Meara, "The Passing Sport Show » The Montreal Daily Star, 6 août 1936, p. 24.

Voir aussi «Les Américains ne sont pas populaires » Le Canada, 6 août 1936, p. 11 et «La balourdise teutonne ", L'Autorité, 8 août 1936, p. 1.

24. Zotique Lespérance, « Nouvelles et commentaires sportifs », La Patrie, 13 août 1936, p. 21.

25. Zotique Lespérance, loc. cit., 16 août 1936, p. 21.

26. Traduction: [Il y a de la colère chez les compétiteurs européens de souche continentale envers la suprématie d'Owens et cie qui s'est manifestée dans les commentaires éditoriaux, mais les sportifs qui ne connaissent ni race, ni croyances, ni couleur, mais sont plutôt intéressés par la performance, ont accueilli l'exploit de la légion ténébreuse à la manière bien connue du monde sportif] Baz O’Meara, « The Passing Sport Show », The Montreal Daily Star, 8 août 1936, p.8.

27. Les périodiques offrent une ouverture sur le monde. L'attrait pour l'Autre dans le contexte des JO nourrit les stéréotypes, certes, mais permet aussi de les questionner et de penser par exemple le sport comme étant une voie possible vers l'égalité structurelle entre les races (cette remise en question des stéréotypes raciaux reste cependant secondaire). Voir les travaux théoriques d'Hans-Jürgen Lüsebrink sur les notions d'altérité et de figure de l'Autre («La perception de l'Autre : jalons pour une critique littéraire interculturelle », Tangence, $n^{\circ}$ 51, 1996, p. 51-66).

28. Il faudra toutefois attendre 1928 pour que les femmes fassent leur entrée dans les compétitions d'athlétisme.

29. Les données relatives à la présence des femmes aux Olympiques sont tirées du document, «Les femmes dans le Mouvement olympique ", Comité international olympique, 3 juin 2014. Voir note 8 .

30. La natation, l'athlétisme, la gymnastique et l'escrime.

31. «Pas de femmes au village olympique », La Patrie, 2 août 1936, p. 69. 
32. « U.S. Coachs Claim Food Unsuitable », The Montreal Daily Star, 3 août 1936, p. 20.

33. Dennis Chong et James N. Druckman. « Framing theory », Annual Review of Political Science, vol. 10, 2007, p. 103-126.

34. Zotique Lespérance, « Nouvelles et commentaires sportifs », La Patrie, 2 août 1936, p. 53.

35. "Jarrett to confer with Brundage », The Montreal Daily Star, 4 août 1936, p. 19.

36. "Canadians Qualify in Tank Trials - Dutch Girl Captures Free Style Final », The Montreal Daily Star, 10 août 1936, p. 18. "Campbell Steers Uneven Course, Beaten by Scharfer - Dutch Girls Win », The Montreal Daily Star, 14 août 1936, p. 20.

37. La chronique de Cook, portant sur le sport féminin, est présente dans le journal même en dehors des JO. Toutefois, durant les deux semaines de l'événement, elle consacre sa chronique quasi exclusivement aux JO.

38. Laure Bereni, Sébastien Chauvin, Alexandre Jaunait et Anne Revillard, Introduction aux gender studies : manuel des études sur le genre, Bruxelles, De Boeck, 2008.

39. Judith Butler, Trouble dans le genre: Pour un féminisme de la subversion, Paris, La Découverte, $2005,183 \mathrm{p}$

40. « Helen Stephens Nabs Olympic Sprint Title », The Montreal Daily Star , 4 août 1936, p. 19.

41. « Helen Stephens est bien une femme ", L'Illustration nouvelle, 7 août 1936, p. 22.

42. « Potins de Berlin », La Patrie, 6 août 1936, p. 20.

43. Myrtle Cook, « In the Women's Sportlight », The Montreal Daily Star, 6 août 1936, p. 25.

44. Myrtle Cook, «In the Women's Sportlight », The Montreal Daily Star, 8 août 1936, p. 9.

45. Myrtle Cook, « In the Women's Sportlight », The Montreal Daily Star, 12 août 1936, p. 21.

46. Celle-ci prend la parole dans une tribune visible et prestigieuse dans The Montreal Daily Star.

47. Plus un journal s'intéresse aux Jeux, plus cette proportion diminue, allant d'environ $58 \%$ pour Le Canada à $28 \%$ pour le Star.

48. Myrtle Cook, «In the Women's Sportlight », The Montreal Daily Star, 13 août 1936, p. 25.

49. Benedict Anderson, L'imaginaire national, réflexions sur l'origine et l'essor du nationalisme, traduit de l'anglais par Pierre-Emmanuel Dauzat, Paris, La découverte \& Sysos, 2002, p. 55-57. Rappelons que la souveraineté du Canada n'est reconnue par la Grande-Bretagne qu'en 1931.

50. Le drapeau alors désigné comme canadien est le le Red Ensign. Il est rouge et comporte, dans le coin gauche en haut une reproduction de l'Union Jack britannique et des armoiries, dont l'un des quartiers porte trois feuilles d'érable. Il faudra attendre 1965 pour qu'un drapeau, l'Unifolié, soit officiellement adopté au Canada.

51. Benedict Anderson, op. cit., p. 178.

52. Les expressions de La Patrie sont toutes tirées de l'article «Le Canada passe au cinquième rang », La Patrie, 5 août 1936, p. 20.

53. « Winnipeg Boxer Only Canuck Left », The Montreal Daily Star, 11 août 1936, p. 13.

54. « Amyot Tells How He Landed Title », The Montreal Daily Star, 15 août 1936, p. 8.

55. Selon Anderson, la carte du pays, comme plusieurs autres aspects du patrimoine naturel ou culturel d'un territoire, par la reproduction à l'infini grâce au capitalisme de l'imprimé, sont détachés de leur contexte géographique (ou écologique) et deviennent de purs signes nationaux. Benedict Anderson, Op. cit., p. 178.

56. Baz O'Meara, «The Passing Sport Show », The Montreal Daily Star, 10 août 1936, p. 18.

57. «Frank Amyot décroche la première victoire canadienne aux Jeux Olympiques ", Le Canada, 10 août 1936, p. 11

58. « Le Japon gagne la majorité des courses de nage », L'Illustration nouvelle, 10 août 1936, p. 21.

59. «Les nègres remportent une victoire complète à Berlin », L'Illustration nouvelle, 8 août 1936, p.

21

60. Idem.

61. «What Canada did at Olympics Today », The Montreal Daily Star, 3 août 1936, p. 20.

62. « Une victoire olympique pour le Canada », La Patrie, 9 août 1936, p. 66. 
63. «Au panthéon des Sports, Nos Gloires et Leurs Exploits, Le Canada et ses gloires olympiques ", La Patrie, 9 août 1936, p. 68. Les majuscules correspondent à la hiérachisation des éléments du titre.

64. «Les chances du Canada aujourd'hui », Le Canada, 4 août 1936, p. 11.

65. «Ce que le Canada a fait hier aux jeux », Le Canada, 5 août 1936, p. 11.

66. « Le Canada fait belle figure aux Olympiques », L'Illustration nouvelle, 13 août 1936, p. 23.

67. «L'équipe à relais japonaise remport le 800 mètres à relais avec une performance record », Le Canada, 12 août 1936, p. 11.

68. «Orr Flashes Form Reaches 200 final », The Montreal Daily Star, 5 août 1936, p. 19.

69. « Le Canada passe au cinquième rang », La Patrie, 5 août 1936, p. 20.

70. Myrtle Cook, «In the Women's Sportlight », The Montreal Daily Star, 10 août 1936, p. 19.

71. Ce mot est utilisé pour désigner les Canadiens, le plus souvent les Canadiens français, avec une visée qui est souvent offensante lorsqu'il est utilisé par des gens d'autres nationalités. Cook, « In the Women's Sportlight », Ibid., 15 août 1936, p. 9; Ibid., 4 août 1936, p. 20.

72. Myrtle Cook, loc cit., 11 août 1936, p. 17.

73. Traduction libre : L'horizon canadien devient de plus en plus sombre en matière de natation, au moment où nous croyions justement avoir un atout dans la manche du Dominion, nous nous retrouvons le bec à l'eau. Ibid., 13 août 1936, p. 25.

74. Traduction libre : Amyot a prouvé que le Canada a encore des hommes de muscles qui peuvent se tenir debout... Baz O'Meara, "The Passing Sport Show ", The Montreal Daily Star, 10 août 1936, p. 18.

75. Benedict Anderson, op. cit., p. 148-15.

76. «L'équipe de ballon au panier du Canada perd la finale aux mains des Américains », Le Canada , 15 août 1936, p. 11.

77. Benedict Anderson, op. cit., p. 147-148.

78. Ainsi, dans L'Illustration nouvelle, on mentionne, dans le bilan des Jeux du 17 août, les 3 athlètes canadiens Amyot, Loaring et Edwards mais Amyot est présenté comme «le seul champion ».

79. «L'équipe de ballon au panier du Canada...», loc. cit.

80. « Ambroisie », La Patrie, 8 août, p. 37.

81. Benedict Anderson, op. cit., p. 19.

82. " Ottawa fera une réception officielle à Frank Amyot », La Patrie, 11 août 1936, p. 20.

83. « [on demandera à Amyot] l'extraordinaire pagayeur de planter l'Arbre olympique ». Myrtle Cook, «In The Women's Sportlight », 17 août 1936, p. 20.

84. Baz O'Meara, « The Passing Sport Show », 13 août 1936, p. 24.

85. Georges Laferrière, «Le Brasseur futur poids-lourd », La Revue moderne, janvier 1936, p. 25.

86. Horace Lavigne, « Au panthéon des sports », La Patrie, 9 août 1936, p. 68.

87. Myrtle Cook, « In The Women's Sportlight », The Montreal Daily Star, 10 août 1936, p. 19.

88. Myrtle Cook, «In The Women's Sportlight », The Montreal Daily Star, 13 août 1936, p. 25.

89. Myrtle Cook, «In The Women's Sportlight », The Montreal Daily Star, 15 août 1936, p. 9.

90. Baz O’Meara, « The Passing Sport Show », The Montreal Daily Star, 14 août 1936, p. 20.

91. L'expression désigne la riche élite conservatrice, étroitement regroupée autour de ses intérêts propres, qui dirige le Canada entre 1812 et 1841. The Canadian Encyclopaedia,

' http://www.thecanadianencyclopedia.ca/en/article/family-compact/ ${ }^{>}$Page consultée le 8 juin 2015.

92. Traduction libre : [devra parler haut et fort pour convaincre les amateurs que notre équipe se méritait leur pamoison] Baz O'Meara, loc. cit., 13 août 1936, p. 25.

93. Baz O'Meara, loc. cit., 14 août 1936, p. 20. 
94. Notre traduction: [En rappelant constamment au public que le Canada doit obtenir une meilleure équipe pour les prochains Jeux olympiques]. Baz O'Meara, loc. cit., 13 août 1936, p. 24.

95. Horace Lavigne, loc. cit.

96. Elmer Ferguson, "The Gist And Jest of It », Montreal Daily Herald, 4 août 1936, p. 7. La perception de Ferguson ne correspond pas aux résultats de nos analyses.

97. Baz O'Meara, loc. cit., 11 août 1936, p. 16.

98. Horace Lavigne, loc. cit.

99. « d'infrastructures sportives du sport amateur ». Baz O’Meara, loc. cit., 11 août 1936, p. 16.

100. «Les Américains sont favoris », La Patrie, 2 août 1936, p. 68.

101. Henry McLemore, "Claim U.S.A. Should Give Up Marathon », The Montreal Daily Star, 10 août 1936, p. 18 ; Elmer Ferguson, " The Gist and Jest Of It », The Montreal Herald, 13 août 1936, p. 9.

102. Henry McLemore, « Only Miracle Can Keep U.S. From Top », The Montreal Daily Star, 8 août 1936, p. 8.

103. Henry McLemore, « Claim U.S.A. Should Give Up Marathon », loc. cit.

104. Henry McLemore, « Many Thrills In Five Ring Sport Circus », The Montreal Daily Star, 17 août 1936, p. 19.

105. Baz O'Meara, loc. cit., 4 août 1936, p. 19.

106. Ibid.

107. 107. Ibid.

108. Zotique Lespérance, « Nouvelles et commentaires sportifs », La Patrie, 16 août 1936, p. 76.

109. Myrtle Cook, loc. cit., 15 août 1936, p. 9.

\section{ABSTRACTS}

Le sport est depuis toujours un facteur de hiérarchisation des groupes sociaux. Dans une perspective de rapport de pouvoir, il sert tout autant à réaffirmer les diverses relations inégalitaires entre les groupes à l'intérieur d'une société qu'à poser ou illustrer les relations conflictuelles entre nations. Le présent texte explore la façon dont les journaux participent à l'interprétation et à la construction de ces rapports dans le cadre de la couverture des Jeux olympiques de 1936. L'analyse aborde d'abord la question de la race, particulièrement la « mise en valeur " des athlètes noirs. On découvre ainsi que, paradoxalement, le racisme ambiant permet la mise en place d'un débat de fond sur la question. La réflexion se poursuit ensuite avec les rapports de genre en montrant comment les journaux marginalisent les accomplissements des sportives afin d'éviter une remise en question des schémas traditionnels. Finalement, les Olympiades sont aussi l'occasion pour les journaux de construire un récit national fort à travers diverses stratégies discursives. À bien des égards, les Jo permettent de construire une identité canadienne qui cherche à transcender les régionalismes. L'omniprésence de l'expression de ces rapports de pouvoir offre néanmoins aux journalistes l'occasion de réfléchir à ces questions.

\section{INDEX}

Mots-clés: nationalisme, genre, race, sport, Montréal, Jeux olympiques, 1936, Québec, sport féminin 


\section{AUTHORS}

\section{CAMILLE CARON BELZILE}

Camille Caron Belzile est étudiante en maîtrise en littératures de langue française à l'Université de Montréal. Publications : «Le voyage de soi à l'Autre : un générateur d'intrigues dans l'écriture de voyage du Nouveau Monde ", Actes du colloque des Rendez-vous de la recherche émergente, CRILCQ, Édition 2015, (accepté pour publication).

\section{EVE LÉGER-BÉLANGER}

Eve Léger-Bélanger est étudiante au doctorat en littératures de langue française à l'Université de Montréal. Publications : « Le corps de la jeune fille au début du XXe siècle : une différenciation marquée selon les auteurs masculins ou féminins? », Les Savoirs des femmes [en ligne], avril 2015. URL : http://savoirdesfemmes.org/public_html/?page_id=161; « La théâtralisation du pâtir dans la pièce de L'Ile de la demoiselle d'Anne Hébert », Actes de colloque Les XXVIIes Journées de la Linguistique [en ligne], Université Laval, mars 2013. URL : http://jdl.lli.ulaval.ca/documents/ actes/actes_2013_Leger-Belanger.pdf ; «Quand conquête terrestre devient conquête sur papier : l'Allemagne dans La Presse en mai 1940 », Micheline Cambron, Alex Gagnon et Myriam Côté (dir.), Les Journaux québécois d'une guerre à l'autre. Deux états de la vie culturelle québécoise au XX ${ }^{e}$ siècle, Québec, Editions Codicille, (sous presse) ; «L'Allemagne dans La Presse et la littérature québécoise de l'entre-deux-guerres : une représentation univoque?», Actes du Colloque International Interdisciplinaire de l'Association des Etudiant-e-s Diplômé-e-s du Département d'histoire de l'Université de Montréal, Conflits et sociétés : des armes aux discours (mai 2014), Université de Montréal, Cambridge Scholars Publishing, directeur du dossier : Nari Shelekpayev (accepté pour la publication) ; « Paradis d'Abdulrazak Gurnah : une culture de l'échange », Actes du colloque international (E)migrations, transferts, exils :métissages et dynamiques de la ville (avril 2015), Université Masaryk de Brno (République Tchèque), Etudes romanes de Brno (soumis) ; « Fascination et catégorisation : construction de l'espace et du lieu liés à l'Allemagne dans la presse et la littérature québécoises de l'entre-deux-guerres ", Actes du colloque Parcourir, cartographier, habiter : les lieux en littérature - Colloque étudiant du Département des littératures de langue française de l'Université de Montréal (janvier 2015), Sens public [en ligne] (soumis).

\section{ALEX GIROUX}

Alex Giroux est étudiant en maitrise en histoire à l'Université du Québec à Montréal.

\section{MARILOU ST-PIERRE}

Marilou St-Pierre est candidate au doctorat en communication à l'Université Concordia. Publications : Charron Jean, Marilou St-Pierre et Geneviève Drolet. « La parole des femmes dans les journaux télévisés au Québec, 1961-2010 », Communication [En ligne], vol. 33/1 | 2015, mis en ligne le 16 février 2015. URL: http://communication.revues.org/5127; DOI: 10.4000/ communication.5127; « Women Sports Reporters: Feminity in a traditional male field ». Dans II International Conference Gender and Communication, Conference proceeding, 2014, p. 50-63.

\section{MICHELINE CAMBRON}

Micheline Cambron est professeure au Département des littératures de langue française de l'Université de Montréal et membre du Centre de recherche interuniversitaire sur la littérature 
et la culture québécoises (CRILCQ). Elle consacre ses recherches et son enseignement à la littérature et à la culture québécoises des $\mathrm{XIX}^{\mathrm{e}}$ et $\mathrm{XX}^{\mathrm{e}}$ siècles. Elle a principalement travaillé sur les formes de l'utopie, les relations presse/littérature et les questions d'histoire littéraire et culturelle. Elle est également spécialiste des œuvres de Fernand Dumont et Paul Ricœur.

\section{DOMINIQUE MARQUIS}

Dominique Marquis est professeure d'histoire à l'Université du Québec à Montréal. Spécialiste d'histoire de la presse québécoise et d'histoire socioreligieuse du Québec, elle a publié plusieurs articles sur la presse au Québec Elle a participé au projet Histoire du livre et de l'imprimé au Canada / History of the Book in Canada. Elle est l'auteure de Un quotidien pour l'Église : L'Action catholique, 1910-1940, paru en 2004 aux Éditions Leméac. Elle mène actuellement un projet de recherche sur Jules-Paul Tardivel et $L a$ Vérité, un réseau ultramontain au service d'une œuvre. Elle est membre du groupe de recherche "La presse montréalaise et la vie culturelle dans l'entre-deux-guerres » et du Groupe de recherches et d'études sur le livre au Québec (GRELQ). 